\title{
Torsionally induced exciton localization and decoherence in $\pi$-conjugated polymers
}

Cite as: J. Chem. Phys. 149, 214107 (2018); https://doi.org/10.1063/1.5054176

Submitted: 30 August 2018 . Accepted: 20 November 2018 . Published Online: 06 December 2018

William Barford (D), and Jonathan R. Mannouch

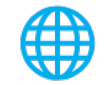

\section{ARTICLES YOU MAY BE INTERESTED IN}

Ultra-fast relaxation, decoherence, and localization of photoexcited states in $\pi$-conjugated polymers

The Journal of Chemical Physics 148, 034901 (2018); https://doi.org/10.1063/1.5009393

Perspective: Computational chemistry software and its advancement as illustrated through three grand challenge cases for molecular science

The Journal of Chemical Physics 149, 180901 (2018); https://doi.org/10.1063/1.5052551

The symmetrical quasi-classical approach to electronically nonadiabatic dynamics applied to ultrafast exciton migration processes in semiconducting polymers

The Journal of Chemical Physics 149, 044101 (2018); https://doi.org/10.1063/1.5037815

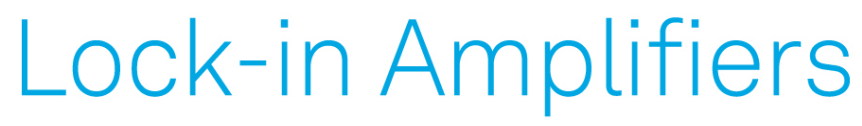

... and more, from DC to $600 \mathrm{MHz}$

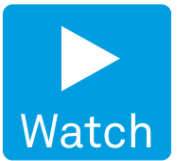




\title{
Torsionally induced exciton localization and decoherence in $\pi$-conjugated polymers
}

\author{
William Barford ${ }^{1,2}$ and Jonathan R. Mannouch ${ }^{1,3}$ \\ ${ }^{1}$ Department of Chemistry, Physical and Theoretical Chemistry Laboratory, University of Oxford, \\ Oxford OX1 3QZ, United Kingdom \\ ${ }^{2}$ Balliol College, University of Oxford, Oxford OX1 3BJ, United Kingdom \\ ${ }^{3}$ University College, University of Oxford, Oxford OX1 4BH, United Kingdom
}

(Received 30 August 2018; accepted 20 November 2018; published online 6 December 2018)

\begin{abstract}
We develop a model of excitons coupled to the rotational motion of monomers to study the torsionally induced relaxation and decoherence of excitons in $\pi$-conjugated polymers. The model assumes that the monomer units are described by elastically uncoupled harmonic oscillators and that there is a linear exciton-roton coupling. Although the rotational degrees of freedom are much slower than the exciton, so that the adiabatic approximation is generally expected to be valid, we also investigate possible quantized roton corrections via coupled time evolving block decimation-Ehrenfest equations of motion. For the relaxation of the lowest-excited exciton, we find that (1) for a polymer chain with a ground state spiral torsional conformation, the equilibrium angular displacement of each monomer is proportional to the difference of the exciton bond-orders on the neighboring bridging bonds. Consequently, this displacement vanishes in the long chain limit and a classical (Landau) exciton-polaron is not formed. (2) For a polymer chain with a ground state staggered torsional conformation, the equilibrium angular displacement of each monomer is proportional to the sum of the exciton bond-orders on the neighboring bridging bonds. Consequently, there is significant angular displacement and local planarization causing exciton density localization. A classical (Landau) exciton-polaron is formed where the staggered angular displacement is proportional to the exciton density. (3) Generally, in the adiabatic limit, the decay of off-diagonal long-range order (i.e., exciton decoherence) mirrors the localization of the exciton density. However, quantum corrections to the rotational motion alter this adiabatic prediction because of correlated exciton-roton dynamics within the first rotational half-period. In particular, exciton-polaron quasiparticle formation causes more rapid and oscillatory exciton decoherence and slower exciton density localization. Published by AIP Publishing. https://doi.org/10.1063/1.5054176
\end{abstract}

\section{INTRODUCTION}

Various mechanisms contribute to the relaxation, decoherence, and localization of photoexcited states (i.e., excitons) in $\pi$-conjugated polymers. The fastest process (occurring on time scales of tens of $\mathrm{fs}$ ) is associated with the coupling of the exciton to high-frequency $\mathrm{C}-\mathrm{C}$ bond vibrations. As described in an earlier paper, ${ }^{1}$ this coupling leads to an ultrafast ( $\left.\leq 10 \mathrm{fs}\right)$ decay in the off-diagonal long-range order of the exciton reduced density matrix (i.e., to rapid exciton decoherence). The cause of this decoherence is the rapid establishment of shortrange exciton-phonon correlations. Importantly, Ref. 1 showed that because the exciton-polaron quasiparticle ${ }^{2}$ thus formed has a relatively light effective mass, this self-trapping is not associated with dynamical self-localization [i.e., to classical (Landau) exciton-polaron ${ }^{2}$ formation]. ${ }^{3-5}$

Another mechanism of exciton relaxation occurs via coupling to the torsional modes of the monomers around their bridging bonds, occurring on time scales of $\sim(0.1-1)$ ps. Since the angular rotations are much slower than $\mathrm{C}-\mathrm{C}$ bond vibrations, they behave more "classically." The following questions then arise: What is the mechanism of decoherence associated with exciton-torsional coupling? Is this associated with exciton density localization (i.e., to Landau polaron formation)? What are the associated experimental observables?

We address these questions in this paper by developing a model of exciton-rotational dynamics. We apply this to a model polymer, namely, poly(para-phenylene) (PPP), where the rotational dynamics are phenyl-ring dynamics. Since we are interested in possible corrections to a classical treatment of the rotational degrees of freedom, we quantize the rotational oscillators. The model is solved via a recently developed time evolving block decimation (TEBD)-Ehrenfest method. ${ }^{6}$ We find that for polymers with a ground state staggered torsional conformation, classical (Landau) exciton-polarons are formed, there is local planarization, and, to a reasonable approximation, exciton decoherence is associated with exciton density localization. However, rotational quantum fluctuations do affect the dynamics, leading to exciton-roton correlations which enhance exciton decoherence while inhibiting exciton localization. By contrast, we find a negligible role of excitonroton coupling for polymers with a ground state spiral torsional conformation.

Assuming classical rotational modes, torsionally induced exciton relaxation and localization have been modelled by a number of authors, ${ }^{7,8}$ while a recent quantum mechanical treatment of torsional modes was reported in Ref. 9. 
In Sec. II, we develop our model and describe the theoretical techniques to solve it. Section III describes our results, and we conclude in Sec. IV.

\section{MODEL AND NUMERICAL TECHNIQUES}

\section{A. Model Hamiltonian of exciton-roton dynamics}

In order to develop a tractable model of coupled excitonroton dynamics, it is necessary to make a number of simplifying assumptions. Taking PPP as our model system, first, we assume that each phenyl ring executes simple harmonic motion about its ground state equilibrium position, $\phi_{n}^{0}$, where $\phi_{n}^{0}$ is the rotational angle around the local molecular axis joining neighboring rings. Second, we assume that there is no elastic coupling between the phenyl rings. These two approximations mean that the torsional motion of each ring is modelled as an Einstein oscillator, described by the rotational angle $\phi_{n}$ and its angular momentum, $L_{n}$. Third, we assume that the angular displacement of each ring, $\Delta \phi_{n}=\left(\phi_{n}-\phi_{n}^{0}\right)$, from its ground state equilibrium value is small. This approximation allows us to derive a linear coupling between the exciton and roton degrees of freedom. The Hamiltonian thus derived [see Eq. (8)] is equivalent to the Frenkel-Holstein model which describes an exciton coupled to $\mathrm{C}-\mathrm{C}$ bond stretches. ${ }^{1}$

If the photoexcited states are Frenkel excitons with a transfer integral, $J$, between neighboring rings, the first two assumptions described above lead to the following Frenkel-Roton Hamiltonian:

$$
\hat{H}=\sum_{n=1}^{N-1} J\left(\theta_{n}\right) \hat{T}_{n, n+1}+\sum_{n=1}^{N} \varepsilon_{n} \hat{N}_{n}+\sum_{n=1}^{N}\left(\frac{K}{2}\left(\Delta \phi_{n}\right)^{2}+\frac{L_{n}^{2}}{2 I}\right) .
$$

In this equation, the "site" index, $n$, labels a phenyl ring, $N$ is the number of rings, $K$ is the elastic force constant, $I$ is the moment of inertia of each ring, and

$$
\hat{T}_{n, n+1}=\left(\hat{a}_{n+1}^{\dagger} \hat{a}_{n}+\hat{a}_{n}^{\dagger} \hat{a}_{n+1}\right)
$$

is the bond-order operator, where $\hat{a}_{n}^{\dagger}\left(\hat{a}_{n}\right)$ creates (destroys) a Frenkel exciton on ring $n$.

The exciton transfer integral,

$$
J\left(\theta_{n}\right)=J_{D D}+J_{S E} \cos ^{2} \theta_{n},
$$

has two components: the through-space dipolar term, $J_{D D}$, and the through-bond superexchange term, $J_{S E}$. It is the superexchange term that results in exciton-roton coupling through its dependence on the bond torsional angle, $\theta_{n}=\left(\phi_{n+1}-\phi_{n}\right)$.

The second term in Eq. (1) describes a Frenkel exciton localized on the $n$th ring, with excitation energy $\varepsilon_{n}$. Since we are assuming uniform chains and thus constant $\varepsilon_{n}$, this term will now be neglected as it does not affect the dynamics.

We now linearize the exciton-roton coupling by assuming small angular displacements in the excited state from the ground state equilibrium conformation, i.e., $\Delta \phi_{n} \ll 1$. This implies that

$$
J\left(\theta_{n}\right) \simeq J_{n}^{0}-J_{S E} \sin 2 \theta_{n}^{0} \times\left(\Delta \phi_{n+1}-\Delta \phi_{n}\right),
$$

where

$$
J_{n}^{0}=J_{D D}+J_{S E} \cos ^{2} \theta_{n}^{0} .
$$

Introducing dimensionless variables

$$
\tilde{\phi}=(K / \hbar \omega)^{1 / 2} \phi
$$

and

$$
\tilde{L}=(\omega / \hbar K)^{1 / 2} L
$$

gives

$$
\begin{aligned}
\hat{H}= & \sum_{n=1}^{N-1}\left[J_{n}^{0}-\hbar \omega A\left(\theta_{n}^{0}\right)\left(\Delta \tilde{\phi}_{n+1}-\Delta \tilde{\phi}_{n}\right)\right] \hat{T}_{n, n+1} \\
& +\frac{\hbar \omega}{2} \sum_{n=1}^{N}\left(\left(\Delta \tilde{\phi}_{n}\right)^{2}+\tilde{L}_{n}^{2}\right)
\end{aligned}
$$

where

$$
A\left(\theta_{n}^{0}\right)=\frac{J_{S E} \sin 2 \theta_{n}^{0}}{(\hbar \omega K)^{1 / 2}}
$$

is the dimensionless exciton-roton coupling constant and $\omega=(K / I)^{1 / 2}$ is the rotational angular frequency.

Finally, we quantize the canonically conjugate variables $\Delta \tilde{\phi}_{n}$ and $\tilde{L}_{n}$ by introducing the harmonic oscillator raising and lowering operators, $\hat{b}_{n}^{\dagger}$ and $\hat{b}_{n}$. Thus, setting

$$
\Delta \tilde{\phi}_{n} \rightarrow \Delta \hat{\tilde{\phi}}_{n}=\frac{1}{\sqrt{2}}\left(\hat{b}_{n}^{\dagger}+\hat{b}_{n}\right) \equiv \hat{B}_{n}
$$

and

$$
\tilde{L}_{n} \rightarrow \hat{\tilde{L}}_{n}=\frac{i}{\sqrt{2}}\left(\hat{b}_{n}^{\dagger}-\hat{b}_{n}\right),
$$

the Hamiltonian becomes

$$
\begin{aligned}
\hat{H}= & \sum_{n=1}^{N-1}\left[J_{n}^{0}-\hbar \omega A\left(\theta_{n}^{0}\right)\left(\hat{B}_{n+1}-\hat{B}_{n}\right)\right] \hat{T}_{n, n+1} \\
& +\hbar \omega \sum_{n=1}^{N}\left(\hat{b}_{n}^{\dagger} \hat{b}_{n}+\frac{1}{2}\right)
\end{aligned}
$$

Equation (10) defines the operator $\hat{B}_{n}$ corresponding to the dimensionless angular displacement.

Before describing the computational technique for simulating the dynamics, let us first consider the classical (adiabatic) limit of this model. Using Eq. (8), the torque on each ring is

$$
\Gamma_{n}=-\frac{\partial\langle\hat{H}\rangle}{\partial \Delta \tilde{\phi}_{n}}=-\Delta \tilde{\phi}_{n}+\lambda_{n},
$$

where, for convenience later, we define

$$
\lambda_{n}=A\left(\theta_{n-1}^{0}\right)\left\langle\hat{T}_{n-1, n}\right\rangle-A\left(\theta_{n}^{0}\right)\left\langle\hat{T}_{n, n+1}\right\rangle .
$$

Setting $\Gamma_{n}=0$ gives the equilibrium angular displacements in the excited state as

$$
\Delta \tilde{\phi}_{n}^{\mathrm{eq}}=\lambda_{n} .
$$

The significance of the form of Eq. (14) will be discussed in Sec. III A. 


\section{B. Dynamics}

As discussed in Sec. IV, ultrafast processes will relax the photoexcited state onto a chromophore (i.e., a local exciton ground state (LEGS) ${ }^{10,11}$ ). Thus, for the torsionally induced relaxation, we may assume that the initial state is an exciton in its lowest excited state with all the rotational oscillators in their ground state,

$$
|\Psi(t=0)\rangle=\left(\frac{2}{N+1}\right)^{1 / 2} \sum_{n=1}^{N} \sin \left(\frac{\pi n}{N+1}\right) \hat{a}_{n}^{\dagger}|\mathrm{GS}\rangle .
$$

As the simulation proceeds, the coupling of the exciton to the oscillators displaces them from their ground state equilibrium values, which means that higher energy levels of the oscillators are occupied. In principle, the number of oscillator excitations is unbounded and the Hilbert space grows exponentially with chain size. For a fixed and small number of oscillator excitations per site (or ring), this exponential growth can be truncated via the time evolving block decimation (TEBD) method, briefly described in Sec. II B 1. However, for large displacements and/or a small oscillator frequency, the number of occupied levels is not small. Indeed, the number of oscillator excitations exceeds

$$
n=\frac{1}{2}\left((\Delta \phi)^{2}\left(\frac{K}{\hbar \omega}\right)-1\right)
$$

For the parameters used in these simulations (see Sec. II C), $n \gtrsim 3$, which is too many for an efficient use of the TEBD method. Thus, in Sec. II B 2, we describe the TEBD-Ehrenfest method, which — unlike the Ehrenfest method—retains quantum correlations between the fast (i.e., excitonic) degree of freedom and the slow (i.e., harmonic oscillator) degrees of freedom, but which is accurate for only one or two roton excitations per site.

\section{Time evolving block decimation (TEBD)}

Given the initial conditions, the state of the system is propagated in time via the evolution operator, i.e.,

$$
|\Psi(t)\rangle=\exp (-i \hat{H} t / \hbar)|\Psi(t=0)\rangle .
$$

We perform this procedure numerically using the Tensor Network Theory (TNT) library, ${ }^{12}$ which implements the time evolving block decimation (TEBD) method. ${ }^{13}$ Details of the TEBD method are described in Appendix B of Ref. 1. Briefly, the TEBD method works by (1) representing $|\Psi\rangle$ as a matrix product state, ${ }^{14}$ (2) expressing the evolution operator via a Trotter decomposition, and (3) compressing the action of the evolution operator on $|\Psi\rangle$ via a singular value decomposition. Time-dependent expectation values of relevant observables are determined via $|\Psi(t)\rangle$.

\section{TEBD and Ehrenfest}

The TEBD-Ehrenfest method was devised by Mannouch for dynamical simulations of the Frenkel-Holstein model in the adiabatic regime. An outline derivation is described in the Appendix; full details for the Frenkel-Holstein model will be published shortly. ${ }^{6}$
We assume that

$$
\Delta \hat{\tilde{\phi}}_{n}=\Delta \tilde{\phi}_{n}^{\mathrm{eh}}+\delta \hat{\tilde{\phi}}_{n},
$$

where $\Delta \tilde{\phi}_{n}^{\text {eh }}$ is the mean-field displacement subject to the Ehrenfest equations of motion,

$$
\begin{gathered}
\frac{d \Delta \tilde{\phi}_{n}^{\mathrm{eh}}}{d \tilde{t}}=\tilde{L}_{n}^{\mathrm{eh}}, \\
\frac{d \tilde{L}_{n}^{\mathrm{eh}}}{d \tilde{t}}=\Gamma_{n}=-\Delta \tilde{\phi}_{n}^{\mathrm{eh}}+\lambda_{n}-\gamma \tilde{L}_{n}^{\mathrm{eh}},
\end{gathered}
$$

and $\tilde{t}=\omega t . \delta \hat{\tilde{\phi}}_{n}$ is the quantum fluctuation around the mean-field displacement, $\Delta \tilde{\phi}_{n}^{\text {eh }}$, whose expectation value will generally be small in the adiabatic regime.

$\lambda_{n}$ is defined in Eq. (14) and is calculated using the instantaneous $|\Psi(t)\rangle$. For generality, we have also included a phenomenological damping term to Eq. (21), which models dissipative interactions with the environment. The purpose of introducing this damping term is to ensure that the dynamical variables achieve their equilibrated values within reasonable simulation times. However, we also note that in the under-damped regime, damping reduces the intrinsic angular frequency to

$$
\omega^{\prime}=\left(\omega^{2}-\gamma^{2} / 4\right)^{1 / 2} .
$$

Equations (20) and (21) are numerically integrated using the Verlet algorithm.

The time-evolution of $|\Psi(t)\rangle$ is numerically determined using Eq. (18) via the TEBD method, where $\hat{H}$ is now replaced by an effective Hamiltonian, defined by

$$
\begin{aligned}
\hat{H}_{\text {eff }}= & \sum_{n=1}^{N-1}\left[J_{n}^{0}-\hbar \omega A\left(\theta_{n}^{0}\right)\left(\Delta \tilde{\phi}_{n+1}^{\mathrm{eh}}-\Delta \tilde{\phi}_{n}^{\mathrm{eh}}\right)\right] \hat{T}_{n, n+1} \\
& -\hbar \omega \sum_{n=1}^{N-1} A\left(\theta_{n}^{0}\right)\left(\hat{B}_{n+1}^{\prime}-\hat{B}_{n}^{\prime}\right)\left(\hat{T}_{n, n+1}-\left\langle\hat{T}_{n, n+1}\right\rangle\right) \\
& +\hbar \omega \sum_{n=1}^{N}\left(\hat{b}_{n}^{\prime} \hat{b}_{n}^{\prime}+\frac{1}{2}\right) \\
& +\frac{\hbar \omega}{2} \sum_{n=1}^{N-1} A\left(\theta_{n}^{0}\right)\left(\Delta \tilde{\phi}_{n+1}^{\mathrm{eh}}-\Delta \tilde{\phi}_{n}^{\mathrm{eh}}\right)\left\langle\hat{T}_{n, n+1}\right\rangle .
\end{aligned}
$$

Here,

$$
\hat{B}_{n}^{\prime}=\frac{1}{\sqrt{2}}\left(\hat{b}_{n}^{\dagger}+\hat{b}_{n}^{\prime}\right) \equiv \delta \hat{\tilde{\phi}}_{n}
$$

and $\hat{b}_{n}^{\prime \dagger}$ is the displaced harmonic oscillator creation operator given by Eq. (A8).

\section{Model parameters}

Model parameters for poly(para-phenylene) are: exciton transfer integrals, $J_{S E}=-1.5 \mathrm{eV}$ and $J_{D D}=-0.5 \mathrm{eV}$; elastic force constant ${ }^{8} K=4 \mathrm{eV} \mathrm{rad}^{-2}$ and moment of inertia of a phenyl ring ${ }^{15} I \sim 10^{26} \mathrm{eV} \mathrm{s}^{2}$, implying that $\hbar \omega=20 \mathrm{meV}$ with a rotational period of $T=2 \pi / \omega=0.21$ ps. For a ground state spiral conformation, we take $\phi_{n}^{0}=n \times 20^{0}$ and $\theta_{0}=20^{\circ}$, while for a ground state staggered conformation, we take $\phi_{n}^{0}=(-1)^{n} \times 10^{0}$ and $\theta_{0}=(-1)^{n} \times 20^{\circ}$. We set $\gamma=\omega / 2$. 


\section{RESULTS}

\section{A. Adiabatic limit}

We first describe our predictions in the classical (i.e., adiabatic, $\hbar \omega \rightarrow 0$ ) limit. The static angular displacements in the excited state are determined by solving Eq. (15) iteratively via the Hellmann-Feynman method. The results for $\Delta \phi_{n}$ for a ground state spiral conformation are shown in Fig. 1. We note that as the chain size increases, the amplitude of the displacement decreases while the width of the displacement increases. In the long chain limit, the displacement vanishes. By contrast, the results for a ground state staggered conformation are displayed in Fig. 2. Here we note that the amplitude and width of the displacement saturate at $N \sim 20$, while the inset shows that the chain is almost completely planarized over $\sim 4$ monomers.

The distinction between a ground state staggered or spiral torsional conformation can be understood via Eqs. (14) and (15). For a uniform ground state staggered conformation, $A\left(\theta_{n-1}^{0}\right)=-A\left(\theta_{n}^{0}\right)$ because $\theta_{n-1}^{0}=-\theta_{n}^{0}$, and thus the equilibrium angular displacement of the $n$th ring is proportional to the sum of the bond-order expectation values of the neighboring bridging bonds. Furthermore, the sign of $\lambda_{n}$ alternates as a function of $n$. Conversely, for a uniform ground state spiral conformation, $A\left(\theta_{n-1}^{0}\right)=A\left(\theta_{n}^{0}\right)$ because $\theta_{n-1}^{0}=\theta_{n}^{0}$, and thus the equilibrium angular displacement of the $n$th ring is proportional to the difference of the bond-order expectation values of the neighboring bonds. Thus, as confirmed by the numerical results, a polymer with a staggered ring conformation undergoes considerably more planarization than a polymer with a spiral ring conformation. This difference can be understood physically as follows. In a staggered conformation, planarization of the bond torsional angles occurs if neighboring rings rotate in a counterclockwise direction (i.e., they rotate towards each other as $\Delta \tilde{\phi}_{n}^{\text {eq }}$ is staggered). For a spiral conformation, however, if two neighboring rings rotate towards each other to reduce the bond torsional angle $\theta_{n}$, the neighboring torsional angles, $\theta_{n-1}$ and $\theta_{n+1}$, increase. Since this motion is not compatible for all bonds, the planarization is restricted.

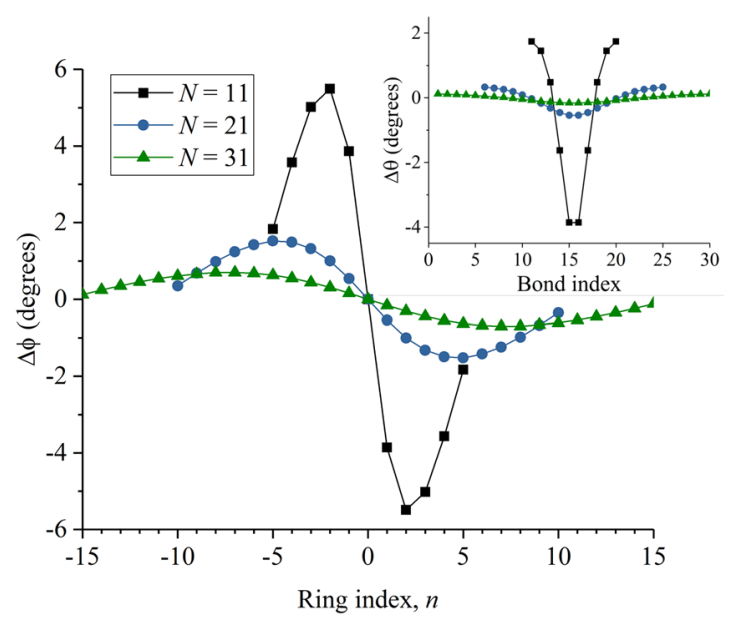

FIG. 1. The angular displacement, $\Delta \phi_{n}$, as a function of ring index for different chain lengths, $N$, for a ground state spiral conformation in the classical (adiabatic) limit. The inset shows the torsional displacement, $\Delta \theta_{n}=\left(\Delta \phi_{n+1}\right.$ $\left.-\Delta \phi_{n}\right)$, for the same chain lengths.

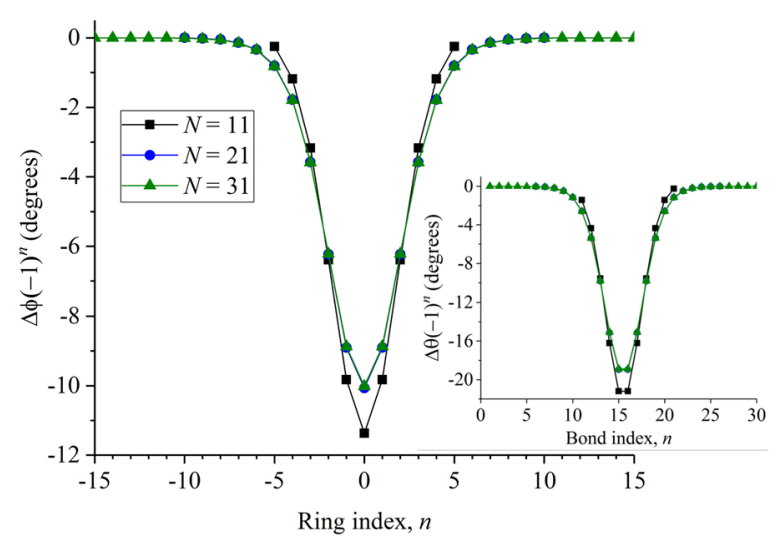

FIG. 2. The staggered angular displacement, $\Delta \phi_{n} \times(-1)^{n}$, as a function of ring index for different chain lengths, $N$, for a ground state staggered conformation in the classical (adiabatic) limit. The inset shows the staggered torsional displacement, $\Delta \theta_{n} \times(-1)^{n}=\left(\Delta \phi_{n+1}-\Delta \phi_{n}\right)$, for the same chain lengths.

In the continuum limit, it is easy to show from Eq. (15) that for a ground state spiral conformation,

$$
\Delta \tilde{\phi}_{n} \sim \psi_{n} \frac{d \psi_{n}}{d n},
$$

while for a ground state staggered conformation,

$$
\Delta \tilde{\phi}_{n} \sim\left|\psi_{n}\right|^{2} \times(-1)^{n},
$$

where $\psi_{n}$ is the exciton center-of-mass wavefunction. Equation (26) is the same condition on the displaced angular variable as for the displaced normal mode in the Holstein model, for which self-localized Landau polarons are known to be the solutions in the adiabatic limit. ${ }^{16,17}$

From now we only consider ground state staggered conformations. We next consider the dynamics of exciton localization and decoherence in the classical (adiabatic) limit. This is achieved by numerically integrating the Ehrenfest equations of motion [Eqs. (20) and (21)] and propagating the state vector using only the first term in the Hamiltonian, Eq. (23) (where now $\Delta \tilde{\phi}_{n}^{\text {eh }} \equiv \Delta \tilde{\phi}_{n}$ as $\left\langle\delta \tilde{\phi}_{n}\right\rangle$ vanishes).

We define the exciton localization length as the spread of the exciton density via

$$
L_{\mathrm{loc}}=5.6\left(\left\langle n^{2}\right\rangle-\langle n\rangle^{2}\right)^{1 / 2},
$$

where

$$
\langle x\rangle=\sum_{m}\left\langle x_{m} \hat{N}_{m}\right\rangle
$$

and the prefactor of 5.6 guarantees that $L_{\mathrm{loc}}=N$ for a particlein-a-box wavefunction in the continuum limit. ${ }^{11,18}$ By contrast, the coherence length is a measure of off-diagonal long-range order in the exciton density matrix ${ }^{19,20}$ and is defined via

$$
L_{\mathrm{coh}}=\sum_{m, n}\left|\left\langle\hat{a}_{m}^{\dagger} \hat{a}_{m+n}\right\rangle\right| .
$$

Figure 3 illustrates $L_{\mathrm{coh}}$ and $L_{\mathrm{loc}}$ as a function of time for different chain lengths. Both of these length scales have nearly decayed to their equilibrated values within one torsional period (i.e., $\sim 200 \mathrm{fs}$ ). Moreover, their values have converged for chains lengths of $N \sim 20$. Apart from very early times, these length scales are proportional to each other, implying 


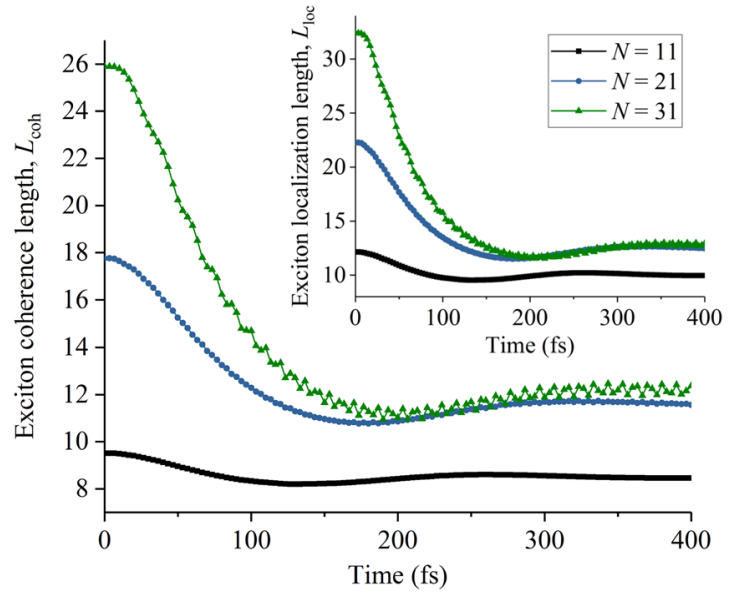

FIG. 3. The time-evolution of the exciton coherence length, $L_{\text {coh }}$ [defined in Eq. (29)] and (inset) the exciton localization length, $L_{\text {loc }}$ [defined in Eq. (27)], for different chain lengths in the adiabatic limit for a ground state staggered conformation.

that in the adiabatic limit exciton localization and decoherence are both associated with classical (Landau) exciton-polaron formation.

\section{B. Quantum regime}

Previous work has shown that it is necessary to quantize the high frequency $\mathrm{C}-\mathrm{C}$ bond vibrations to correctly explain the mechanism of exciton decoherence. ${ }^{1}$ Although rotational modes are much slower than vibrational modes, it is also possible that quantizing them leads to additional exciton decoherence mechanisms.

We first establish that the TEBD-Ehrenfest solution of the fully quantized model, Eq. (12), has converged with the number of roton excitations per site, $N_{\text {ro }}$. Figure 4 shows the staggered angular displacement, $\left\langle\Delta \phi_{n}\right\rangle \times(-1)^{n}$, for a chain length $N=21$ for different values of $N_{\text {ro }}$. The simulation has

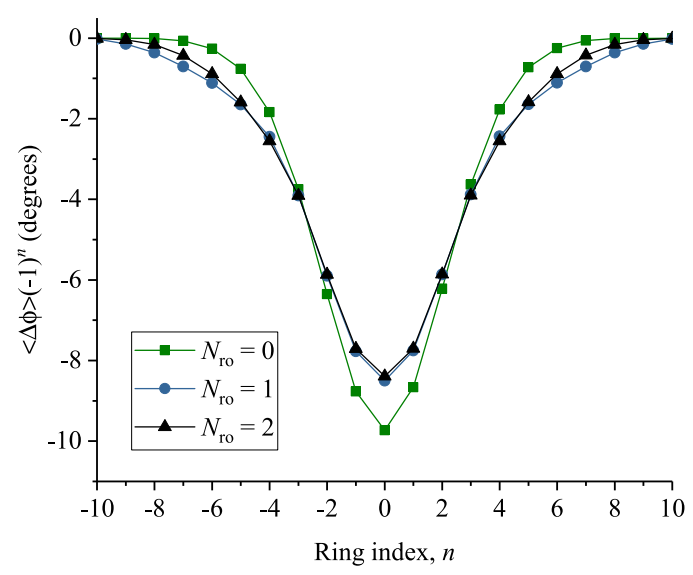

FIG. 4. The equilibrated (taken at $t=400 \mathrm{fs} \sim 2 T$ ) staggered angular displacement, $\left\langle\Delta \phi_{n}\right\rangle \times(-1)^{n}$, as a function of ring index for different numbers of roton excitations per site, $N_{\text {ro }}\left[N_{\text {ro }}=0\right.$ is the classical (adiabatic) limit of the quantum model]. $N=21$ with a ground state staggered conformation.

converged by $N_{\text {ro }}=2$ (a value we use for the remaining calculations). We also note that quantum fluctuations in the rotational angles slightly reduce the amplitude and increase the width of the angular displacements.

The time-evolution of the staggered angular displacement, $\left\langle\Delta \phi_{n}\right\rangle \times(-1)^{n}$, is shown in Fig. 5, illustrating that these displacements have reached their equilibrated values (as shown in Fig. 4) after two torsional periods (i.e., $t \gtrsim 400 \mathrm{fs}$ ). The inset also displays the time-evolution of the exciton density, $\left\langle N_{n}\right\rangle$, showing exciton density localization after a single torsional period ( $200 \mathrm{fs}$ ) (see also the inset of Fig. 7). As predicted by Eq. (26), in the long-time limit (i.e., $t \gtrsim 400$ fs), $\left\langle\Delta \phi_{n}\right\rangle \propto\left\langle N_{n}\right\rangle$ $\times(-1)^{n}$, illustrating classical (Landau) polaron formation.

So far we have focused on classical (Landau) polaron formation, where the non-linear coupling of the exciton bondorder to the angular variables causes self-localization of the exciton such that the exciton density mirrors the staggered angular displacement. We now focus on our predictions for

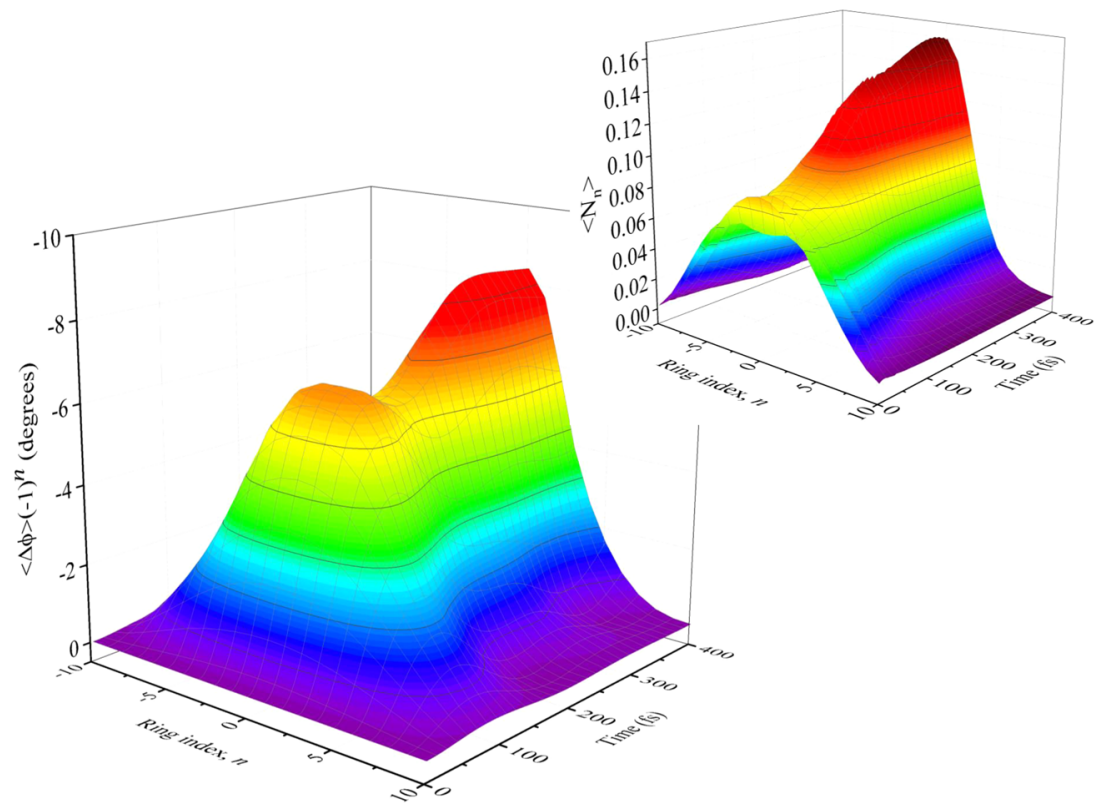

FIG. 5. The time-evolution of the staggered angular displacement, $\left\langle\Delta \phi_{n}\right\rangle$ $\times(-1)^{n}$, for a chain size $N=21$. The inset displays the time-evolution of the exciton density, $\left\langle N_{n}\right\rangle$, showing exciton density localization after a single torsional period $(\sim 200 \mathrm{fs})$. In the long-time limit (i.e., $t \gtrsim 400 \mathrm{fs}$ ), $\left\langle\Delta \phi_{n}\right\rangle \propto\left\langle N_{n}\right\rangle$ $\times(-1)^{n}$, illustrating classical (Landau) polaron formation. 


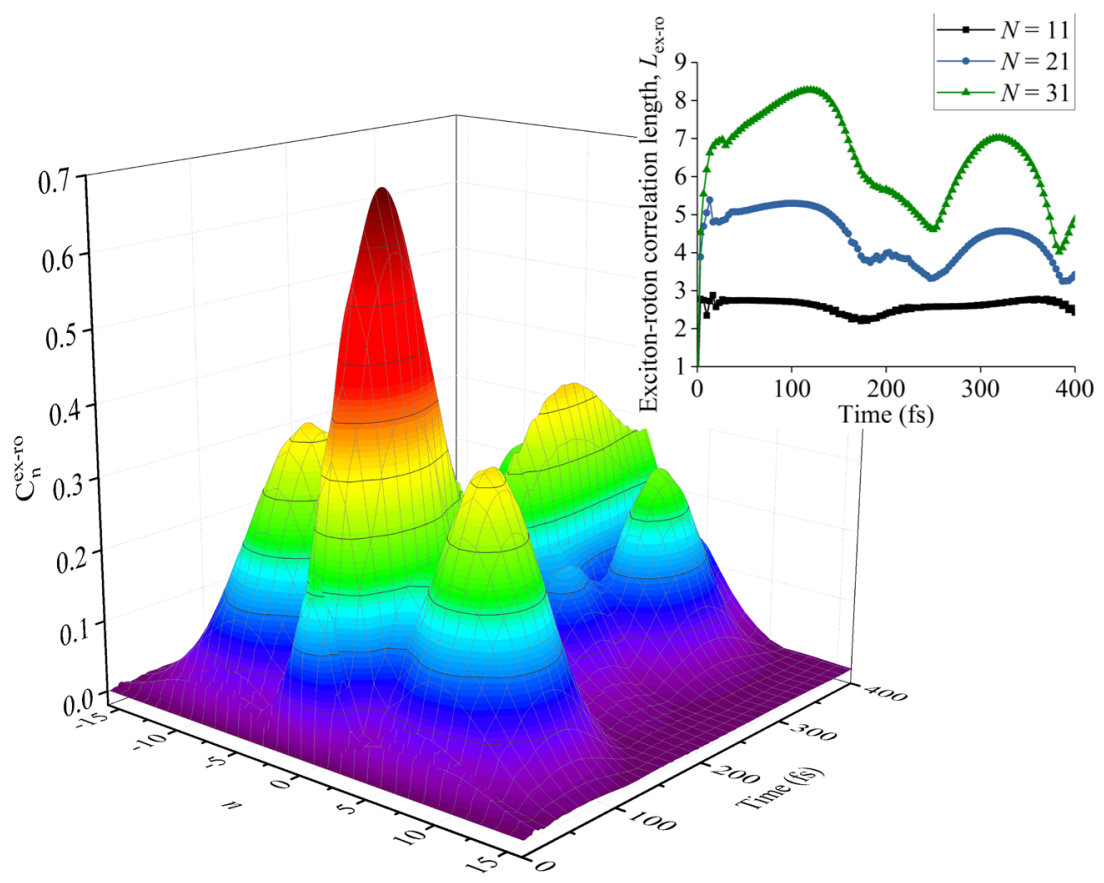

FIG. 6. The time-evolution of the exciton-roton correlation function, $C_{n}^{\text {ex-ro }}$ [defined in Eq. (30)], for a chain size $N=21$. The inset shows the exciton-roton correlation length, $L_{\text {ex }}$-ro [defined in Eq. (31)], for different chain lengths.

exciton-polaron quasiparticle formation, i.e., the correlation of the exciton with a roton $n$ monomers away. This correlation is quantified by the staggered exciton-roton correlation function defined by

$$
C_{n}^{\text {ex-ro }}=\sum_{m}(-1)^{n}\left\langle\hat{N}_{m} \hat{B}_{m+n}^{\prime}\right\rangle,
$$

where $\hat{N}_{m}$ is the exciton number operator and $\hat{B}_{m+n}^{\prime}$, defined in Eq. (24), is the quantized angular displacement (relative to the mean-field displacement) $n$ rings away from the instantaneous exciton position.

As shown in Fig. 6, exciton-roton correlations are established very rapidly and are maximized at half a rotational period (i.e., at $t=100 \mathrm{fs}$ ); they subsequently gradually decay as a function of time. The staggered exciton-roton correlation function, $C_{n}^{\text {ex-ro }}$, is an even function of $n$ and shows secondary maxima. In the adiabatic limit (i.e., $\hbar \omega \rightarrow 0$ ), the excitonroton correlation length diverges because the rotons respond infinitely slowly to the exciton. ${ }^{3,5}$ Since $J / \hbar \omega \sim 100$, the rotons in this model are in the adiabatic regime and we would expect a large exciton-roton correlation length. The inset of Fig. 6 shows the exciton-roton correlation length, defined as

$$
L_{\text {ex-ro }}=\frac{\sum_{n}\left|n C_{n}^{\text {ex-ro }}\right|}{\sum_{n}\left|C_{n}^{\text {ex-ro }}\right|},
$$

for different chain lengths. Evidently, for the chain lengths considered here, $L_{\text {ex -ro }}$ has not converged.

Finally, we address the question as to what are the experimental observables associated with exciton-polaron quasiparticle formation. Both two-dimensional spectroscopy and fluorescence depolarization are related to off-diagonal longrange order of the exciton density matrix, $\left\langle\hat{a}_{m}^{\dagger} \hat{a}_{m+n}\right\rangle,{ }^{1}$ and thus to the exciton coherence length, $L_{\mathrm{coh}}$.

Figure 7 show the exciton coherence length, $L_{\mathrm{coh}}$, calculated from both the quantum model and its classical (adiabatic) limit. For times less than half a rotational period, we observe that $L_{\mathrm{coh}}$ decays more rapidly in the quantum model and thence undergoes oscillations commensurate with the torsional period. The more rapid decay of $L_{\mathrm{coh}}$ in the quantum model can be understood by expressing it as ${ }^{1}$

$$
L_{\mathrm{coh}}=\sum_{m, n}\left|\psi_{m} \psi_{m+n}^{*}\left\langle\Phi_{m+n} \mid \Phi_{m}\right\rangle\right| .
$$

Equation (32) shows that $L_{\mathrm{coh}}$ is proportional to the overlap of the exciton probability amplitudes as well as to the overlap of the roton state vectors, $\left|\Phi_{m}\right\rangle$. Because of the finite range of the exciton-roton correlation in the quantum model, these rotational overlaps decay with distance as the exciton-roton correlation is established, leading to another decay mechanism for exciton coherences.

The inset of Fig. 7 shows the exciton localization length, $L_{\text {loc }}$. In contrast to $L_{\text {coh }}$, this decays more slowly in the

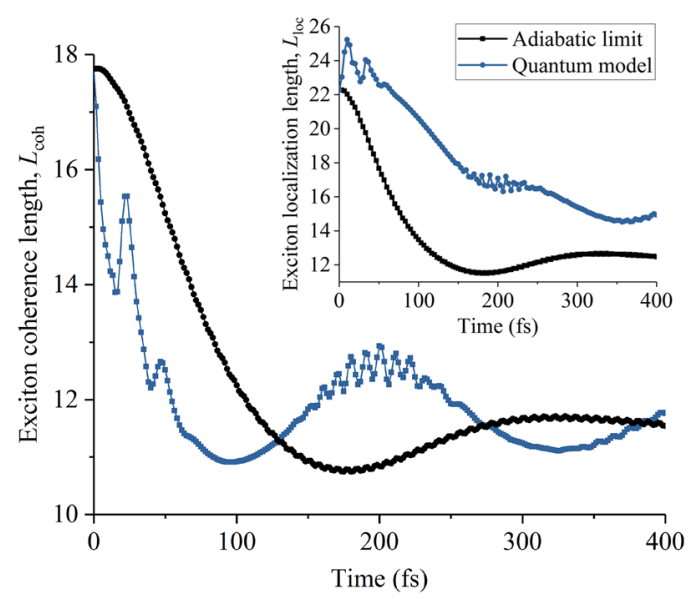

FIG. 7. The time-evolution of the exciton coherence length, $L_{\mathrm{coh}}$ [defined in Eq. (29)], and (inset) the exciton localization length, $L_{\text {loc }}$ [defined in Eq. (27)], for the quantum model and in the adiabatic limit. The chain length $N=21$. 
quantum model because quantized rotational fluctuations reduce the amplitude of the classical (Landau) polaron and thus reduce exciton density localization.

\section{CONCLUDING REMARKS}

We have developed a model of excitons coupled to the rotational motion of monomers to study the torsionally induced relaxation and decoherence of excitons in $\pi$-conjugated polymers. The model assumes that the monomer units are described by elastically uncoupled harmonic oscillators and that there is a linear exciton-roton coupling. Although the rotational degrees of freedom are much slower than the exciton, so that the adiabatic approximation is generally expected to be valid, we also investigate possible quantized roton corrections via coupled TEBD-Ehrenfest equations of motion. For the relaxation of the lowest-excited exciton, we find that:

1. For a polymer chain with a ground state spiral torsional conformation, the equilibrium angular displacement of each monomer is proportional to the difference of the exciton bond-orders on the neighboring bridging bonds. Consequently, this displacement vanishes in the longchain limit and a classical (Landau) exciton-polaron is not formed.

2. For a polymer chain with a ground state staggered torsional conformation, the equilibrium angular displacement of each monomer is proportional to the sum of the exciton bond-orders on the neighboring bridging bonds. Consequently, there is significant angular displacement and local planarization causing exciton density localization. A classical (Landau) exciton-polaron is formed where the staggered angular displacement is proportional to the exciton density.

3. Generally, in the adiabatic regime, the decay of offdiagonal long-range order (i.e., exciton decoherence) mirrors the localization of the exciton density. However, quantum corrections to the rotational motion alter this adiabatic prediction because of correlated exciton-roton dynamics within the first rotational half-period. In particular, exciton-polaron quasiparticle formation causes more rapid and oscillatory exciton decoherence and slower exciton density localization.

The torsional relaxation times reported in this paper ( $\sim 0.4 \mathrm{ps}$ ) are determined by our choice of parameters (namely, an intrinsic torsional period of $0.2 \mathrm{ps}$ and a damping time of $0.4 \mathrm{ps}$ ). Although sub-ps torsional dynamics have been observed, ${ }^{21,22}$ typically longer torsional relaxation times ${ }^{23,24}$ of ca. 10 ps are observed experimentally, which we attribute to over-damping of torsional modes in solution or side chains on rings increasing their moment of inertia.

In this paper, we have made the distinction between ideal ground state spiral and staggered conformations and predicted the planarization in the long-chain limit. In practice, however, polymer chains are subject to both static and dynamic torsional fluctuations, meaning that polymer chains are never in an ideal ground state spiral or staggered conformations and that the exciton is always localized onto finite-length chromophores.
Using the results of this and our earlier paper, ${ }^{1}$ we can now summarize the key intra-polymer dynamical processes affecting photoexcited states (broadly similar conclusions are reached in Ref. 9). Hot excitations undergo ultra-fast relaxation and decoherence because of coupling to quantized $\mathrm{C}-\mathrm{C}$ bond vibrations. The excitons do not exhibit density localization from this mechanism because the phonon exciton-polaron quasiparticle has a relatively small effective mass. As shown in Ref. 1, however, dissipative interactions with the environment cause the extended (delocalized) exciton wavefunctions to "collapse" (or localize) onto chromophores (i.e., into local exciton ground states $\left.{ }^{11,25}\right)$. As shown in this paper, torsional modes also cause exciton decoherence, which initially is associated with exciton-roton correlations, but for longer time scales is associated with density localization. Exciton density localization now occurs because the roton exciton-polaron quasiparticle has a relatively large effective mass. ${ }^{5}$ Assuming that environmental coupling has already "collapsed" the exciton wavefunction onto a chromophore, this localization will occur on the chromophore and will be observed via fluorescence depolarization. ${ }^{26}$

Equations (14) and (15) indicate that ring planarization is a consequence of the torsional modes coupling directly to the exciton bond-order [see also the second term of Eq. (8)]. This mechanism differs from others (see, e.g., Ref. 9) where the exciton density couples to a normal mode displacement. In future work, we intend to supplement our model by such a coupling (as described in Ref. 1). We will also investigate the combined roles of vibrational and rotational degrees of freedom, disorder, and, in particular, the effect of driven rotational fluctuations which are known to cause exciton dynamics. ${ }^{15,27}$

\section{ACKNOWLEDGMENTS}

W.B. would like to thank Professor Jianshu Cao and the Department of Chemistry at MIT for hospitality during an academic visit sponsored by the Balliol-MIT Academic Exchange Program. J.R.M. would like to thank the EPSRC Centre for Doctoral Training, Theory and Modelling in Chemical Sciences (under Grant No. EP/L015722/1) and University College Oxford (through a Radcliffe Scholarship) for financial support. This work was performed using the Tensor Network Theory Library, Beta Version 1.2.1 (2016), S. Al-Assam, S. R. Clark, D. Jaksch, and the TNT Development team, www.tensornetworktheory.org. We thank Michael Lubasch for help in using the TNT Library.

\section{APPENDIX: TEBD-EHRENFEST DYNAMICS}

The assumption underlying an efficient implementation of the TEBD-Ehrenfest method is that the quantum fluctuations, $\left\langle\delta \tilde{\phi}_{n}\right\rangle$, of the harmonic oscillators around their meanfield displaced values, $\Delta \tilde{\phi}_{n}^{\text {eh }}$, are small. $\Delta \tilde{\phi}_{n}^{\text {eh }}$ is subject to the Ehrenfest equations of motion, while $\left\langle\delta \tilde{\phi}_{n}\right\rangle$ is determined by the solution of an effective Hamiltonian, $\hat{H}_{\text {eff }}$, that we seek to derive.

In general, the state vector for a quantum system described by Eq. (12) can be written in the form 


$$
|\Psi\rangle=\sum_{j} \sum_{\{p\}} C_{j,\{p\}}(t)|j,\{p(t)\}\rangle,
$$

where $C_{j,\{p\}}(t)$ is a time-dependent coefficient determined by $\hat{H}_{\text {eff }}$ and $|j,\{p(t)\}\rangle$ is a time-dependent basis that follows the Ehrenfest mean-field trajectory. In particular,

$$
|j,\{p(t)\}\rangle=\left|\psi_{j}\right\rangle\left|p_{1}(t)\right\rangle\left|p_{2}(t)\right\rangle \cdots\left|p_{N}(t)\right\rangle,
$$

where $\left|\psi_{j}\right\rangle$ is an eigenstate of the Frenkel-exciton Hamiltonian [i.e., the first two terms in Eq. (1)].

The ket $\left|p_{n}(t)\right\rangle$ is the $p$ th displaced excitation of the coherent state on the $n$th site, $\left|\alpha_{n}\right\rangle$, i.e.,

$$
\left|p_{n}(t)\right\rangle=\frac{\left(b_{n}^{\prime \dagger}\right)^{p}}{\sqrt{p !}}\left|\alpha_{n}\right\rangle
$$

where

$$
\left|\alpha_{n}(t)\right\rangle=\exp \left(-\left|\alpha_{n}\right|^{2} / 2\right) \sum_{q} \frac{\alpha_{n}^{q}}{\sqrt{q !}}\left|q_{n}\right\rangle
$$

and

$$
\left|q_{n}\right\rangle=\frac{\left(b_{n}^{\dagger}\right)^{q}}{\sqrt{q !}}|0\rangle .
$$

$\left|\alpha_{n}\right\rangle$ is parameterized by the "displacement"

$$
\alpha_{n}(t)=\frac{\Delta \tilde{\phi}_{n}^{\mathrm{eh}}+i \tilde{L}_{n}^{\mathrm{eh}}}{\sqrt{2}}
$$

which satisfies the Ehrenfest equations of motion [i.e., Eqs. (20) and (21)],

$$
\frac{d \alpha_{n}}{d t}=-i \alpha_{n}+i \frac{\lambda_{n}}{\sqrt{2}}
$$

where $\lambda_{n}$ is defined in Eq. (14).

In Eq. (A3), $b_{n}^{\prime \dagger}$ is the instantaneously displaced harmonic oscillator creation operator, defined with respect to the undisplaced harmonic oscillator creation operator via

$$
b_{n}^{\prime \dagger}=\left(b_{n}^{\dagger}-\alpha_{n}^{*}(t)\right)
$$

The requirement that $|\Psi\rangle$ is subject to the time-dependent Schrödinger equation

$$
i \hbar \frac{d|\Psi\rangle}{d t}=\hat{H}|\Psi\rangle,
$$

where $\hat{H}$ is defined in Eq. (12), implies that $C_{j,\{p\}}(t)$ is subject to the equation of motion

$$
i \hbar \frac{d C_{j,\{p\}}(t)}{d t}=\sum_{j^{\prime}} \sum_{\left\{p^{\prime}\right\}}\left\langle j,\{p\}\left|\hat{H}_{\mathrm{eff}}\right| j^{\prime},\left\{p^{\prime}\right\}\right\rangle C_{j^{\prime},\left\{p^{\prime}\right\}}(t) .
$$

Time-differentiating Eq. (A1) and using Eq. (A3) to Eq. (A8) gives an expression for $\hat{H}_{\text {eff }}$, i.e., Eq. (23).

${ }^{1}$ J. R. Mannouch, W. Barford, and S. Al-Assam, J. Chem. Phys. 148, 034901 (2018).

2 "We define an exciton-polaron quasiparticle as an exciton dressed by a cloud of quantized harmonic oscillators (e.g., phonons or rotons)." Although "self-trapped" in a translationally invariant system, this quasiparticle forms a Bloch state. ${ }^{3}$ Conversely, we define a classical (Landau) exciton-polaron ${ }^{4}$ as a self-trapped particle which breaks translational symmetry. A Landau exciton-polaron is thus "self-localized" with an associated static harmonic oscillator displacement field.

${ }^{3}$ O. R. Tozer and W. Barford, Phys. Rev. B 89, 155434 (2014).

${ }^{4}$ L. D. Landau, Phys. Z. Sowjetunion 3, 884 (1933).

${ }^{5}$ W. Barford, M. Marcus, and O. R. Tozer, J. Phys. Chem. A 120, 615 (2016).

${ }^{6}$ J. R. Mannouch, "Exciton dynamics in $\pi$-conjugated polymers," $\mathrm{Ph} . \mathrm{D}$. thesis, University of Oxford, 2019.

${ }^{7}$ I. Franco and S. Tretiak, J. Am. Chem. Soc. 126, 12130 (2004).

${ }^{8}$ W. Barford, I. Boczarow, and T. Wharram, J. Phys. Chem. A 115, 9111 (2011).

${ }^{9}$ R. Binder, D. Lauvergnat, and I. Burghardt, Phys. Rev. Lett. 120, 227401 (2018); R. Liang, S. J. Cotton, R. Binder, R. Hegger, I. Burghardt, and W. H. Miller, J. Chem. Phys. 149, 044101 (2018).

${ }^{10}$ V. Malyshev and V. A. Malyshev, Phys. Rev. B 63, 195111 (2001).

${ }^{11}$ D. V. Makhov and W. Barford, Phys. Rev. B 81, 165201 (2010).

${ }^{12}$ S. Al-Assam, S. R. Clark, and D. Jaksch, J. Stat. Mech.: Theory Exp. 2017, 093102.

${ }^{13}$ G. Vidal, Phys. Rev. Lett. 91, 147902 (2003).

${ }^{14}$ U. Schollwöck, Ann. Phys. 326, 96 (2011).

${ }^{15}$ O. R. Tozer and W. Barford, J. Chem. Phys. 143, 084102 (2015).

${ }^{16}$ T. Holstein, Ann. Phys. 8, 325 (1959); 8, 343 (1959).

${ }^{17}$ E. I. Rashba and M. D. Sturge, Excitons (Elsevier Science North-Holland, Amsterdam, 1982).

${ }^{18} \mathrm{An}$ essentially equivalent measure of exciton density localization is defined by Eq. (23) of Ref. 1. See, F. C. Spano, S. C. J. Meskers, E. Hennebicq, and D. Beljonne, J. Chem. Phys. 129, 024704 (2008).

${ }^{19}$ O. Kuhn and V. Sundstrom, J. Chem. Phys. 107, 4154 (1997).

${ }^{20}$ C. Smyth, F. Fassioli, and G. D. Scholes, Philos. Trans. R. Soc., A 370, 3728 (2012).

${ }^{21}$ N. P. Wells and D. A. Blank, Phys. Rev. Lett. 100, 086403 (2008).

${ }^{22}$ J. Clark, T. Nelson, S. Tretiak, G. Cirmi, and G. Lanzani, Nat. Phys. 8, 225 (2012).

${ }^{23}$ S. Westenhoff, W. J. D. Beenken, R. H. Friend, N. C. Greenham, A. Yartsev, and V. Sundström, Phys. Rev. Lett. 97, 166804 (2006).

${ }^{24}$ P. Parkinson, C. Müller, N. Stingelin, M. B. Johnston, and L. M. Herz, J. Phys. Chem. Lett. 1, 2788 (2010).

${ }^{25}$ M. Marcus, O. R. Tozer, and W. Barford, J. Chem. Phys. 141, 164102 (2014).

${ }^{26}$ A. Ruseckas, P. Wood, I. D. W. Samuel, G. R. Webster, W. J. Mitchell, P. L. Burn, and V. Sundström, Phys. Rev. B 72, 115214 (2005).

${ }^{27}$ N. M. Albu and D. Yaron, J. Phys. Chem. C 117, 12299 (2013). 